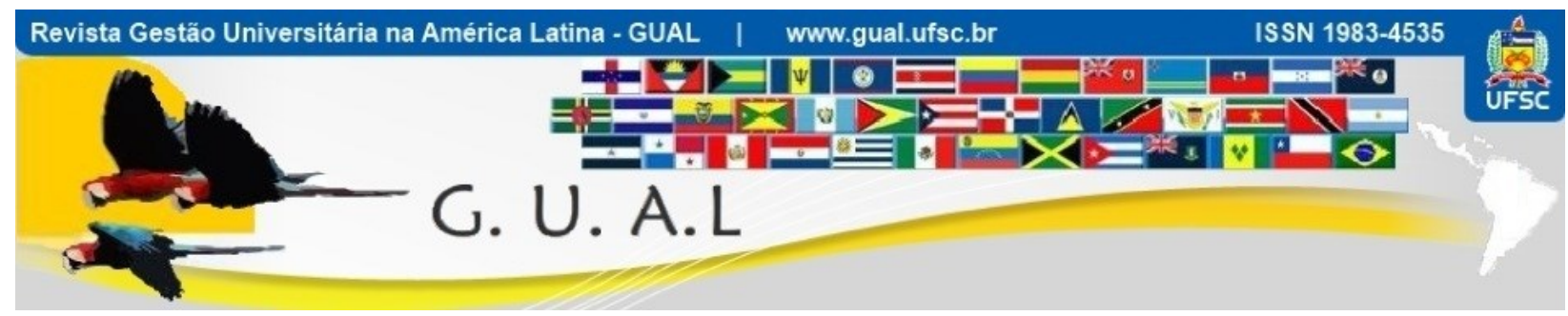

DOI: http://dx.doi.org/10.5007/1983-4535.2015v8n4p153

\title{
ÉTICA EN LA INVESTIGACIÓN CIENTÍFICA, EN EL ÁREA DE LAS CIENCIAS SOCIALES, EN UNIVERSIDADES DEL PARAGUAY
}

\section{THE ETHICS OF SOCIAL SCIENCE RESEARCH AT PARAGUAYAN UNIVERSITIES}

Marta Isabel Canese de Estigarribia, Doutora Universidad Tecnológica Intercontinental mcanese@gmail.com

Recebido em 05/dezembro/2014

Aprovado em 05/abril/2015

Sistema de Avaliação: Double Blind Review

Esta obra está sob uma Licença Creative Commons Atribuição-Uso. 


\title{
RESUMEN
}

Esta investigación analiza las bases teóricas y principios éticos de la investigación social a fin de aportar al fortalecimiento de las prácticas actuales en las universidades del Paraguay. La metodología tuvo un alcance exploratorio, cualitativo, mediante una revisión bibliográfica y documental de las declaraciones, guías y códigos de ética científica publicados por organizaciones internacionales y nacionales. Los resultados señalan que la preocupación sobre la ética en la investigación científica comenzó en las Ciencias de la Salud, siendo aplicada a toda investigación con personas. A partir de 1980, se cuenta a nivel internacional con guías éticas específicas para la investigación social, que destacan la importancia de la empatía, del diálogo permanente y la negociación con la comunidad participante. Para fortalecer las prácticas éticas en la investigación social en las universidades del Paraguay, este estudio considera que mas allá de los requisitos burocráticos, debe basarse en una ética dialógica y situacional, que promueva una actitud y un proceso de investigación auténticamente ético, respetuoso de la dignidad de las personas, sus diferencias y su autonomía, y que contribuya a la justicia y a la equidad.

Palabras clave: Bases Teóricas. Guía Ética. Investigación Social. Diálogo.

\begin{abstract}
This article analyzes the theoretical and ethical principles of social research to contribute to the strengthening of current practices at the universities in Paraguay. The methodology used had an exploratory scope, following a qualitative approach, through a literature research and documentary analysis, including declarations, guidelines and codes of scientific ethics published by national and international organizations. This analysis indicate that the concern about ethics in scientific research began in the Health Sciences, and later were applied with human subjects and Social Sciences. Since 1980, there are specific ethical guidelines for social research, highlighting the importance of empathy, and the ongoing dialogue and negotiation with the participating community. To strengthen ethical practices in social research at the universities in Paraguay, this study finds that, beyond the bureaucratic requirements, research practices must be based on dialogical and situational ethics to promote an ethical attitude toward the research process, to respect people's dignity, their differences and their autonomy, and contribute to justice and equity.
\end{abstract}

Keywords: Social Research. Theoretical Bases. Ethical Guidance. Dialogue. 


\section{INTRODUCCIÓN}

El desarrollo de nuevos conocimientos en el área de las Ciencias Sociales requiere necesariamente de la participación de las personas y, consecuentemente, el respeto de sus derechos por parte de los investigadores. Si bien, por lo general, toda investigación social manifiesta su intención de beneficiar a la sociedad en la solución de algún problema, es posible que algunos experimentos o estudios observacionales puedan causar inconvenientes, alteraciones o incluso daños a las personas o grupos humanos participantes. En todos los casos, la responsabilidad del investigador social, como persona y como profesional, incluye el respeto a los derechos humanos de estas personas cuyas vidas pueden ser afectadas por la investigación. El escaso conocimiento y observación de los cuidados éticos en la investigación social que se desarrolla en la República del Paraguay, y muy especialmente en las investigaciones realizadas en las universidades públicas y privadas, es el motivo principal del desarrollo de este estudio. Por ser un país con escasa trayectoria de investigación, el tema de la ética en la investigación social aún no integra la cultura académica local.

El presente estudio tuvo como propósito analizar las bases teóricas y principios éticos adoptados por los investigadores a nivel internacional, a fin de contribuir al desarrollo de las estratégicas para la construcción de un sistema de evaluación y revisión de las prácticas de cuidados éticos aplicados a la investigación social desarrollada en las universidades de Paraguay. Con ese propósito, se realizó una investigación bibliográfica y documental, delimitado a la revisión de teorías y autores del área, declaraciones internacionales, sistemas de normas y códigos, así como también la exploración de propuestas, declaraciones y códigos nacionales que contemplen cuidados éticos en la investigación social.

\section{2 ÉTICA EN LA INVESTIGACIÓN CON PERSONAS}

La responsabilidad profesional de la condición de investigador demanda la producción de un producto de valor para la ciencia, no sólo en el sentido práctico o monetario, sino sobretodo en su esencia para el bien de la Humanidad. Esta responsabilidad obliga a pensar en el valor ético tanto del producto de la investigación, sino también de los medios empleados para producirlo y los efectos que ese proceso tuvo para los sujetos involucrados o afectados directa o indirectamente por la investigación. Esta preocupación, presente desde los inicios de las Ciencias Humanas y Sociales, adquiere una importancia decisiva ante la indignación mundial causada por las investigaciones inescrupulosas realizadas en la Segunda Guerra 
Mundial, generando una dinámica de encuentros internacionales en los que se comienza a estructurar un sistema de principios y normas que orientan actualmente a todas las investigaciones en las que intervienen seres humanos.

Pero la preocupación sobre los cuidados éticos que deben ser implementados en la investigación científica se inicia en las Ciencias de la Salud, no en las Ciencias Sociales, configurándose a partir de la convocatoria de los gremios relacionados a la investigación biomédica a la discusión y la elaboración de códigos que regulen estos cuidados. A partir del año 1947, se construye un primer instrumento para atender a los cuidados éticos de protección a las personas en las investigaciones científicas, mediante la enunciación del Código de Nuremberg en 1947. Este código consagra la importancia del ejercicio de la autonomía de los sujetos participantes en una investigación, por medio del consentimiento voluntario del mismo o de su representante legal para el caso de menores de edad y personas que no han alcanzado o han perdido la capacidad necesaria para el ejercicio de dicha autonomía. Este primer código, pensado en principio desde y para las investigaciones del área de salud, obliga además al investigador a informar de forma comprensible a los participantes sobre la naturaleza, los objetivos, los métodos, la duración, los inconvenientes o posibles daños que pudiera causarle una investigación. Señala la libertad de cada sujeto participante de retirarse en el momento que crea conveniente, y que el estudio pueda terminar prematuramente como consecuencia de la autonomía de los participantes.

El pronunciamiento de Helsinki, en 1964, y otros posteriores, amplían los principios éticos a tomar en cuenta en la investigación, incrementando la necesidad de una evaluación independiente del equipo de investigación. Si bien estos principios fueron pensados nuevamente en relación a las investigaciones biomédicas, se extienden a las investigaciones de otras áreas siempre que se realicen con personas. Bajo la tutela y la iniciativa de la Organización Mundial de la Salud (OMS), las demás áreas de la investigación en la que participan seres humanos comienzan a adecuarse a los cuidados y principios establecidos.

\section{GUÍAS Y CÓDIGOS ÉTICOS PARA LA INVESTIGACIÓN SOCIAL}

A partir de 1980, los investigadores sociales iniciaron su propia discusión sobre la responsabilidad ética en la investigación social. De estos encuentros, foros y sus discusiones, surgen posteriormente iniciativas para implementar un marco ético mediante guías específicas a nivel internacional. Los principales códigos creados para esa finalidad son: 


\section{ÉTICA EN LA INVESTIGACIÓN CIENTÍFICA, EN EL ÁREA DE LAS CIENCIAS SOCIALES, EN \\ UNIVERSIDADES DEL PARAGUAY \\ DOI: http://dx.doi.org/10.5007/1983-4535.2015v8n4p153}

1. Guía Ética de la Asociación Nacional para la Práctica de la Antropología (NAPA, 1988)

2. Código de Ética de la Asociación Antropológica Americana (AAA, 1998)

3. Guía ética para las Buenas Prácticas en la Investigación de la Asociación de Antropólogos Sociales de Inglaterra (ASA, 1999)

4. Código de Ética de la Asociación Internacional Sociológica (ISA, 2001)

5. Guía para la Ética en las Ciencias Sociales, Leyes y Humanidades, del Comité Nacional para la Ética en la Investigación de las Ciencias Sociales y Humanidades, de Noruega (NESH, 2001)

6. Recomendaciones para la Toma de Decisiones Éticas y la Investigación en Internet de la AoIR (ESS, 2002)

Estos códigos y guías constituyen importantes referencias para la comunidad de investigadores del tercer milenio a la hora de definir los cuidados éticos a tomar en cuenta en una investigación social. La American Anthropological Association (AAA, 1998) propone una formulación gradual y flexible del proceso de obtención del consentimiento informado para investigaciones del área de las Ciencias Sociales, entendiendo que se trata de un proceso permanente y participativo en la trayectoria de la investigación. El proceso de consentimiento se inicia con el diseño del proyecto y continua a través del diálogo con los participantes, y no requiere un formulario escrito específico: "Es la calidad del consentimiento, y no su formato, lo que resulta relevante" (AAA, 1998, p.3). Estas recomendaciones de la AAA se adaptan muy bien a los estudios de caso o a la investigación-acción, en las que el investigador puede construir una red de confianza y corresponsabilidad con los sujetos participantes. Pero esto no significa que sea posible omitir en todos los casos la documentación del consentimiento, sino que se recomienda registrar las estrategias y acuerdos que se alcanzan progresivamente.

A diferencia de otras áreas de investigación científica, la propia naturaleza de la investigación social hace impredecible el curso de los acontecimientos, aunque se tenga un protocolo bien establecido. Esto hace que el investigador se enfrente con circunstancias en las que debe tomar decisiones, debe optar por registrar o no lo que presencia. Por ese motivo, un primer criterio establecido por los códigos éticos en el área de la investigación social es respetar el compromiso y la voluntad de los participantes. El segundo criterio es el tipo de espacio en el que se realiza la investigación: cuando la observación se realiza en un espacio público se puede registrar los datos obtenidos sin correr el riesgo de infringir el respeto a la privacidad de las personas (NESH 2001, p. 12), y esto se extiende a los medios de comunicación social, como la prensa escrita, la televisión o la radio. En cambio, en el caso de observaciones realizadas en espacios privados, los procedimientos de información y la 
documentación del consentimiento de los participantes deben ser muy rigurosos, a fin de prevenir lesiones a la privacidad, riesgos personales o daños a la imagen de personas o grupos sociales.

Sin embargo, otro factor que determina el riesgo que corre el participante está dado por la propia intervención de la investigación. La introducción de una variable extraña al medio natural en el que se procesa el fenómeno, aunque ocurra en un espacio público, puede generar riesgos adicionales (NESH, 2001). Por ejemplo, una investigación sobre el comportamiento de los hinchas de un equipo deportivo en la cancha (espacio público) puede ser observado sin riesgos para los sujetos participantes siempre y cuando la investigación no introduzca elementos extraños que puedan alterar el desarrollo de los acontecimientos. Si en esas mismas circunstancias, el investigador introduce un símbolo, una pregunta o un comentario irritante para un grupo de hinchas, pueden generarse enfrentamientos o situaciones violentas que pongan en riesgo incluso la integridad física de la población que participa en la investigación de forma directa o indirecta, incluso de aquellos que no han sido debidamente informados ni han firmado consentimiento alguno.

El diálogo, la empatía, la capacidad de observar desde la perspectiva del otro, es el punto de partida para la construcción de una ética dialógica y situacional. Esta propuesta no se reduce a procesos burocráticos, como la firma de un consentimiento informado, sino que se traduce en un diálogo con los participantes que comparten el compromiso de la investigación mediante negociaciones y acuerdos. La denominación situacional obedece a que las decisiones éticas se sitúan en cada contexto singular, tomando en consideración a las personas y los medios de investigación. La sensibilidad del investigador juega un papel crucial en esta propuesta, dado que no se cuenta con un protocolo inicial cerrado, sino que se basa en un proceso de "intercambio comunicativo empático que comprenda y respete las diferencias de las personas" (Kisnerman, 2001, p. 2).

\section{4 ÉTICA EN LA INVESTIGACIÓN VIRTUAL}

La ampliación de posibilidades y herramientas que pueden potencializar y facilitar la actividad del investigador social en internet traen como consecuencia también numerosos riesgos y requieren de cuidados éticos específicos. Los derechos de autoría son mucho mas vulnerables en internet, dada la facilidad de copiar y pegar que tienta a muchos investigadores, y principalmente a los estudiantes universitarios que cuentan con un tiempo 
escaso y trabajan bajo mucha presión. Esto ha impulsado el desarrollo de programas informáticos específicos para detectar estos plagios, se han popularizado, reduciendo hasta cierto punto las ventajas de utilizar este atajo a la hora de realizar un trabajo académico en las universidades.

En las investigaciones de campo, por medio de internet, se cuenta actualmente con diversas herramientas facilitadoras para la aplicación de encuestas, entrevistas, observaciones y otras técnicas de frecuente aplicación en la investigación social. Los principales problemas surgen cuando la investigación se realiza en el campo virtual, que ofrece una ventana privilegiada para la observación y la experimentación a los investigadores sociales. La definición de espacio público, en el campo virtual, no es tan sencilla como parece, en algunos casos puede originar controversias. Cuando la investigación social se realiza en el campo virtual, esa determinación toma como base que espacio público comprende a las interacciones cuyo acceso es público (Estalella \& Ardèvol, 2007). Se da por sentado que los usuarios de esos sitios conocen que su comunicación está abierta a todos, pero no siempre eso ocurre. Desde 1990, estas preocupaciones en relación a la creciente utilización de internet en la investigación social, se viene plasmando en códigos y normas específicas, que intentan prever las diversas situaciones en diversos contexto y circunstancias que puedan afectar a las personas.

En el año 2002 se publica la Guía Ética de la Association of Internet Researchers (AoIR), con el título Ethical Decision-making and Internet Research Recommendations from the AoIR Ethics Working Committe. Esta guía señala que las bases para orientar la decisión ética en la investigación en internet deben buscarse en los principios éticos específicos de cada disciplina, acompañados de una orientación específica de los elementos aportados por internet a la investigación para esa disciplina (ESS 2002). La dificultad radica en que las guías éticas de cada área no prevén algunos elementos o riesgos que se presentan cuando se utiliza internet como medio o como espacio de investigación.

\section{5 ÉTICA EN LA INVESTIGACIÓN CIENTÍFICA, EN LAS UNIVERSIDADES DEL PARAGUAY}

En Paraguay, se crea por primera vez en 1994 el Comité de Ética en Investigación (CEI) del Instituto de Investigaciones en Ciencias de la Salud (IICS), dependiente de la Universidad Nacional de Asunción, a fin de velar por el cumplimiento de las normas éticas de los trabajos científicos. El CEI se rige de acuerdo a las siguientes declaraciones: 
1. Declaración Universal de los Derechos Humanos, de 1948.

2. Declaración Universal sobre Bioética y Derechos Humanos proclamada por la UNESCO, del año 2005.

3. Declaración de Helsinki, de la Asociación Médica Mundial, y sus modificaciones hasta el año 2004.

4. Pautas Éticas Internacionales para la Investigación Biomédica en Seres Humanos, del Consejo de Organizaciones Internacionales de las Ciencias Médicas (CIOMS), en colaboración con la Organización Mundial de la Salud (OMS), del año 2002

5. Guía de Principios Internacionales para Investigaciones Biomédicas que envuelven Animales elaborada por la CIOMS en el año 2007.

Constituye un inicio pionero en el análisis y la evaluación autónoma de los trabajos de investigación, principalmente los estudios relacionados con las investigaciones biomédicas. $\mathrm{Su}$ acción inicia la tarea de estructurar la tarea de desarrollar los cuidados éticos en la investigación con personas, si bien aún su campo no contepla de forma específica a la investigación social y sus particularidades. Los principios que fundamentan la labor del CEI contemplan:

1. Respeto a la dignidad de las personas.

2. Respeto a la salud, la integridad, la privacidad y el bienestar de los participantes.

3. Preservación sostenible de los recursos naturales del ambiente vital de la investigación.

4. Distribución equitativa de los beneficios e inconvenientes de la investigación entre las personas y grupos participantes.

5. Realización de evaluaciones autónomas, competentes y oportunas.

6. Consideración respetuosa hacia las necesidades de salud de la comunidad y del país.

7. Consideración respetuosa de las particularidades culturales, religiosas y sociales de los participantes y el grupo social en el que se realice la investigación.

También en el área de la investigación biomédica, se inician las tareas del Comité de Etica en Investigación del Laboratorio Central de Salud Pública (CEI - LCSP), dependiente del Ministerio de Salud Pública y Bienestar Social de la República del Paraguay. Sus funciones son muy específicas, evalúa los protocolos de investigación desde la perspectiva bioética, considerando los los códigos y pautas internacionales, la metodología de la investigación, la evaluación bioética, a fin de evitar la posibilidad de aprobación de investigaciones que impliquen riesgos para las personas. El CEI basa sus actividades en los siguientes códigos y declaraciones: Código de Nuremberg (1947); Informe Belmont (1974); Código de Helsinki (1964), y el documento del Consejo de Organizaciones Internacionales de 
las Ciencias Médicas CIOMS. El CEI del LCSP basa su labor en los siguientes principios (Chamorro, 2012):

1. Respeto a las personas: a su integridad física, su salud, su dignidad y su bienestar.

2. De beneficencia: traer beneficios para las personas participantes y la comunidad.

3. No maleficencia: no ocasionar perjuicios o malestar a las personas participantes y la comunidad.

4. De Justicia: distribuir con justicia los beneficios de la investigación.

En el año 2012, según Chamorro (2012), el CEI del LCSP gestionó y obtuvo el registro internacional en el Departamento de Salud y Servicios Humanos de USA, vía web, y el registro IRB (Institutional Review Board), otorgado por la OHRP (Office for Human Research Protections).

Siempre en el área de la investigación biomédica, algunas universidades privadas, como la Universidad del Pacífico y la Universidad del Norte, han iniciado la actividad de comités de ética de la investigación, a fin de evaluar los trabajos científicos que se desarrollan en las carreras del área de la Salud. Pero en el área de las Ciencias Sociales y Humanidades, aún no se cuenta con propuestas o iniciativas universitarias que orienten los cuidados éticos de los trabajos de investigación. Las diversas facultades, institutos y centros de la UNA, y de las demás universidades públicas y privadas, aún no contemplan ese aspecto, si bien se han iniciado algunos debates y encuentros sobre el tema.

\section{CONSIDERACIONES FINALES}

Los resultados del análisis realizado en este estudio permiten afirmar que se ha avanzado bastante a nivel internacional en cuanto a la definición de los principios y cuidados éticos a tomar en cuenta en la investigación social. Si bien en un comienzo la conciencia y la acción dirigida a estructurar principios y códigos éticos aceptados internacionalmente partieron de las organizaciones relacionadas con la investigación biomédica, en las últimas dos décadas se ha avanzado en la construcción de principios y códigos específicos para la investigación social.

En Paraguay, en función de los resultados del análisis documental realizado, se puede afirmar que los inicios de la aplicación de los principios y códigos de ética internacionales se están estructurando en la Universidad Nacional de Asunción y otras universidades públicas y privadas, mediante la creación de comités de ética dirigidos a la investigación biomédica, si bien sus funciones se aplican a todas las investigaciones con seres humanos. También revelan 


\section{ÉTICA EN LA INVESTIGACIÓN CIENTÍFICA, EN EL ÁREA DE LAS CIENCIAS SOCIALES, EN \\ UNIVERSIDADES DEL PARAGUAY \\ DOI: http://dx.doi.org/10.5007/1983-4535.2015v8n4p153}

los resultados que aún no se ha comenzado a estructurar la implementación de la ética en la investigación social en las universidades del Paraguay.

Este trabajo de investigación recabó elementos que pueden aportar a la construcción de las bases y principios para la elaboración de un sistema que oriente los trabajos de investigación social que se realizan en las universidades en Paraguay. Se han encontrado algunos principios consagrados en las declaraciones, códigos y guías internacionales, como el respeto a los derechos humanos, a la dignidad de las personas y a su autonomía. Esto implica que el investigador debe informar de forma apropiada a las personas participantes o a su representante legal en el caso de menores de edad y personas que no ejercen dicha autonomía por alguna discapacidad. Y significa también que cada persona tiene el derecho de retirarse de la investigación en cualquier momento. Las orientaciones dirigidas a la investigación social se adhieren a este principio bajo otras perspectivas, considerando que el proceso de consentimiento se inicia con el diseño del proyecto y continua durante toda la trayectoria de la investigación mediante el diálogo del equipo investigador con los participantes y la comunidad involucrada en la investigación social. Esto se justifica porque la propia naturaleza de la investigación social hace que no sea posible prever todos los acontecimientos, y que se deban tomar decisiones durante el proceso de la investigación. Una de esas decisiones concierne al registro de lo observado, sin correr el riesgo de perjudicar la imagen o el bienestar de las personas involucradas. El escenario de la investigación juega en la toma de decisiones un papel crucial, mediante la distinción del tipo de espacio en el que se realiza la investigación: público o privado; y las condiciones de intervención de la investigación que pudieran afectar a las personas.

Para construir una guía ética adecuada para la investigación social en Paraguay, este estudio considera, en primer lugar, que la misma debe fundamentarse en una ética dialógica y situacional, basada en la empatía, la capacidad de escuchar y de situarse en el lugar del otro. Sería muy fácil escribir un código de ética para la investigación social con una larga lista de requisitos formales, pero éstos no tendrían sentido si no reflejan una actitud y un proceso de investigación auténticamente ético, respetuoso de la dignidad de las personas, sus diferencias y su autonomía, que asegure el beneficio y preserve a los participantes de perjuicios y riesgos innecesarios, que contribuya a la justicia y a la equidad en la distribución de los beneficios de la investigación. Por tanto, se recomienda la ampliación de la presente investigación a fin de aportar otros elementos necesarios para la construcción de las bases teóricas y los principios 
que habrán de orientar la elaboración de una guía ética dirigida a la investigación social que se desarrolla en las universidades del Paraguay.

\section{BIBLIOGRAFÍA}

AAA. (1998). Code of Ethics of the American Anthropological Association: American Anthropological Association. En URL: http://www.aaanet.org/committees/ethics/ethcode.htm Fecha de acceso: 20 de agosto de 2014.

ASA. (1999). Ethical Guidelines for Good Research Practice. United Kingdom: Association of Social Anthropology. En URL: http://www.theasa.org/ethics/guidelines.htm

Chamorro, G. (2012).Comité de Ética en Investigación (CEI LCSP) - Research Ethics Committee - Laboratorio Central de Salud Pública. Rev. Salud Pública; Vol. 2 No 2; JulioDiciembre 2012 pág. 37-38. Paraguay, 2012.

ESS (2002). Ethical decision-making and Internet research: Recommendations from the AoIR ethics working committee. Association of Internet Researchers (AoIR). En URL:

www.aoir.org/reports/ethics.pdf Fecha de acceso: 19 de agosto de 2014.

Estalella, A. \& Ardèvol, E. (2007). Ética de campo: hacia una ética situada para la investigación etnográfica de internet . Forum Qualitative Sozialforschung / Forum: Qualitative Social Research, 8(3), Art. 2, http://nbn-resolving.de/urn:nbn:de:0114-fqs070328. Fecha de acceso: 21 de agosto de 2014.

ISA. (2001). Code of Ethics: International Sociological Association. En URL:

http://www.isa-sociology.org/about/isa_code_of_ethics.htm Fecha de acceso: 18 de agosto de 2014.

Kisnerman, N. (2001). Ética: un discurso, o práctica social?. Paidos, Buenos Aires.

NAPA. (1988). NAPA Ethical Guidelines: National Association for the Practice of Anthropology. En URL:

http://www.practicinganthropology.org/about/?section=ethical_guidelines Fecha de acceso: 16 de agosto de 2014.

NESH. (2001). Guidelines for research ethics in the social sciences, law and the humanities. Norway: The National Committee for Research Ethics in the Social Sciences and the Humanities (NESH). 